\title{
Author's Reply to Srinivas: "Pharmacokinetics and Pharmacokinetic/Pharmacodynamic Modeling of Filgotinib (GLPG0634), a Selective JAK1 Inhibitor, in Support of Phase IIB Dose Selection"
}

\author{
Florence Namour ${ }^{1}$
}

Published online: 20 October 2015

(c) The Author(s) 2015. This article is published with open access at Springerlink.com

In response to the letter from Dr. Nuggehally R. Srinivas titled "Comment on: "Pharmacokinetics and Pharmacokinetic/Pharmacodynamic Modeling of Filgotinib (GLPG0634), a Selective JAK1 Inhibitor, in Support of Phase IIB Dose Selection"” (Srinivas NR 2015)

We thank Dr. Srinivas for his interest in the publication and his constructive comments. We welcome the opportunity to clarify and discuss additional information about filgotinib and its active metabolite.

The in vitro characterization of the main enzyme(s) involved in the metabolism of filgotinib revealed that carboxylesterase 2 (CES2) is the main isoform involved in the metabolism of filgotinib and in the formation of its major active metabolite. Of interest, some saturation of human CES2 was noticed in vitro, which was partially compensated by human carboxylesterase 1 (CES1) forming the active metabolite. These data indicate that even in the case of complete inhibition and/or saturation of CES2 in humans, the metabolic elimination of filgotinib would not be blocked and liver CES1 could also form the active metabolite. Of note, as an integral part of the validation of the bioanalytical method, we have shown that filgotinib is stable in whole blood as well as in plasma. These data suggest that blood carboxylesterases are not involved in the

On behalf of the co-authors.

This reply refers to the article available at doi:10.1007/s40262-015-0335-6.

Florence Namour

florence.namour@glpg.com

1 Galapagos SASU, 102 Avenue Gaston Roussel, Romainville, France formation of the active metabolite. Nevertheless, although the plateauing effect in plasma curves of the metabolite could be due to conversion of circulating filgotinib, which escaped the first-pass effect, it could also result from slow elimination of the metabolite itself.

As pointed out by Dr. Srinivas, the metabolite/filgotinib area under the plasma-concentration curve ratio tends to decrease with increasing doses, which could reflect the effect of saturation of the pre-systemic conversion of filgotinib to the active metabolite. However, in the case of saturable pre-systemic conversion, one would have expected the maximum plasma concentration to also be affected. However, over the dose range tested, the metabolite/filgotinib ratio estimated for the maximum plasma concentration was found to be relatively constant after single (2.9 and 2.0) and repeated doses (twice daily: 5.8-7.2 and once daily: 3.0-2.0).

With regard to the simulated JAK1 inhibition, similar responses were obtained after $100 \mathrm{mg}$ twice daily and $200 \mathrm{mg}$ once daily (78.5 vs. $77.6 \%$ ). At these two dosing regimens, the effective exposure (sum of parent and metabolite exposure taking into account the lower potency for the metabolite) over the dosing interval is about twofold higher after $200 \mathrm{mg}$ once daily vs. $100 \mathrm{mg}$ twice daily. This suggests that the average concentration is similar for these two treatment regimens, and is predictive of the mean pSTAT1 inhibition.

To clarify, the concept of a saturable pre-systemic conversion of filgotinib to its active metabolite was investigated in the population pharmacokinetic analysis based on two additional models:

- Publication model: dose-independent pre-systemic filgotinib-metabolite conversion and dose-dependent 
"secondary pathway of elimination" of filgotinib (OFV: 23504).

- Alternative 1: saturation of pre-systemic conversion of filgotinib to metabolite and dose-dependent "secondary pathway of elimination" of filgotinib (OFV: 23490).

- Alternative 2: saturation of pre-systemic conversion of filgotinib to metabolite with no "secondary pathway of elimination" of filgotinib (OFV: 23537).

Including the dose-dependent pre-systemic conversion led to a statistically significant improvement in NONMEM OFV of -14 , corresponding to $p=0.0002$ (alternative 1 compared with the publication model). However, this additional component could not replace the secondary pathway of elimination for filgotinib included in the published model, with the aim to describe the lower thanexpected observed metabolite exposure (change in OFV of +33 , alternative 2 vs. the publication model).

This suggests that incorporation of a saturable pre-systemic conversion of filgotinib to its active metabolite could indeed have been a relevant additional component of the population pharmacokinetic model. However, as acknowledged by Dr. Srinivas and based on the moderate improvement in OFV shown above, this would not have impacted the recommendation for phase IIb dose selection.

\section{Compliance with ethical standards}

Florence Namour is an employee of Galapagos SASU. AbbVie has provided funding to Galapagos for the development of filgotinib.

Open Access This article is distributed under the terms of the Creative Commons Attribution-NonCommercial 4.0 International License (http://creativecommons.org/licenses/by-nc/4.0/), which permits any noncommercial use, distribution, and reproduction in any medium, provided you give appropriate credit to the original author(s) and the source, provide a link to the Creative Commons license, and indicate if changes were made.

\section{References}

Srinivas NR (2015) Comment on: "Pharmacokinetics and pharmacokinetic/pharmacodynamic modeling of filgotinib (GLPG0634), a selective JAK1 inhibitor, in support of phase IIB dose selection" Clin Pharmacokinet. doi:10.1007/s40262-015-0335-6. 